\title{
A Theoretical Framework for Analyzing Multicultural Group Learning
}

\author{
Mohd Hafizan Hashim, Michael L. Hoover \\ McGill University, Canada
}

\begin{abstract}
Conflict is a natural part of any multicultural group learning (McGL) environment. Dealing with conflicts in McGL is not an easy task due to multiplicity of differences such as cultural origins, beliefs, and practices. It is crucial to address the challenges and contradictions among learners who are increasingly diverse due to internationalization in higher education systems, especially for the foreign students who must adapt the local cultural norms of behavior and interactions. The core idea of McGL includes the mutually constituting influences of social interactions in jointly constructed activities across multiple settings and the function of mediating psychological tools. However, how students make these adaptations remains unclear. To address this, the present paper proposes Engeström's Activity System Theory (AST) as a theoretical framework for analyzing student interactions in complex multicultural group learning (McGL) environments. This paper describes a framework for identifying the sources of conflict in McGL and ways to deal with those conflicts. The ultimate goal of this program of research is to facilitate students' ability to work together by using activity systems analysis as an analytical tool to better understand McGL interactions, which will enable instructors and students to productively engage in the coconstruction of knowledge.
\end{abstract}

\section{Introduction}

Interest in the study of how diverse students learn in groups has grown due to increasing multiculturalism and internationalization in higher education. These phenomena have transformed the higher education landscape, requiring that multicultural issues be taken into account in studies of student group learning. Multicultural group learning (McGL) has been conceptualized as a group of learners with diverse backgrounds, races, cultural heritage, languages, ethnicities, religions, gender and gender expression, ancestry and places of origin [1]. In McGL, learners are expected not only to think critically and solve a range of problems, but also to engage in constructive dialog in groups in which learners do not share the same culture. Such crosscultural dialog necessarily produces conflicts and contradictions as learners from different communities bring their own culturally specific elements such as signs, symbols, texts, formulas, graphics and languages.

This paper applies Engeström's [5] Activity System Theory (AST) as a theoretical framework for analyzing the complexity of multicultural group learning (McGL). The goal of this paper is to identify the sources of conflict in McGL and to describe the ways that learners deal with them. This paper justifies the use of activity systems analysis as a theoretical framework for studying the multiple interactions, dialog, and problem-solving strategies that occur given the learners' diversity of cultures, practices and beliefs. Specifically, this paper proposes that AST can be used to understand how diverse learners negotiate (or fail to negotiate) the conflicts and contradictions that occur in McGL interactions.

Vygotsky's learning theory extended Piaget's developmental theory of cognitive abilities (to perceive, process, and make sense of knowledge) by embracing the notion of the socio-cultural. Vygotskian scholars have argued that all learning occurs within a cultural context and involves a complex social interaction that requires an understanding of the relationship between the human mind (how people think) and activity (what people do). Learning, from this socio-cultural perspective, is a process of meaning making (instead of knowledge reception) within a specific socio-cultural context through dialogical processes [12].

Cultural Historical Activity Theory (CHAT) is a theoretical standpoint within the field of educational psychology that began with Lev Vygotsky's work in Russia during the 1920s and 1930s. Educational researchers and practitioners have taken components of CHAT to develop theories such as social constructivism, communities of practice, sociocultural theory, and activity theory [20]. Activity Systems Theory (AST) is a CHAT-based approach developed by Engeström [5] for analyzing object-oriented learning activities that involve interactions among subjects, objects, and communities, through motivation, action, goals, socio-historical context and the consequences of the activity. In this approach, "the unit of analysis in activity systems analysis is the object-oriented activity itself" [20]. Since the late 1980s, Vygotsky’s works have become more influential in North America and Europe as a systematic approach that studies human learning as an interaction-based 
engagement between individuals and their environment [3], [5], [8], [20]. Subsequently, this theoretical paradigm has influenced the development of the field of the Learning Sciences beginning in the late 1990s. Although Vygotsky did not distinguish activity from action in his writing, we adopt his student Leont'ev's [15] construct of activity when discussing the multicultural group learning throughout this paper.

The Learning Sciences are interdisciplinary in nature, combining research on knowledge and skills in the fields of cognitive science, educational psychology, instructional design, computer science, anthropology, and sociology. It aims "to better understand the cognitive and social processes that result in the most effective learning and to use this knowledge to redesign classrooms and other learning environments so that people learn more deeply and more effectively" [17]. The field of Learning Sciences has significantly transformed our approach towards learning: from instructionism to a constructivist approach, and gradually shifted to a sociocultural approach.

With the internationalization of higher education, international students are expected to adapt the local cultural norms of behavior and actively participate in classroom interactions. However, this is not an easy task when there are multiple cultures coming into play, giving rise to conflicting norms and expectations among learners. To resolve these multiple conflicts within such complex learning activities, Engeström proposed the third generation of Activity Theory, a reorientation of CHAT from the individual's higher psychological functions to the collective activity systems that then lead learners "to develop conceptual tools to understand dialogue, multiple perspectives, and networks of interacting activity systems" [5]. This reorientation permits us to focus on questions of diversity in the interactions and dialog among students who come from different cultural traditions. Problems in interactions among learners arise when they bring with them their own cultural elements such as signs, symbols, text, formulas, graphics and languages. What binds a group of learners together is that they share a common goal in the learning activity. In contrast to multicultural learning environments, culture remains invisible in a monoculture learning environment, and instructors tend to pay attention to culture only when different cultural patterns arise in the same learning environment. An AST approach provides the means to systematically analyze student interaction while considering how the individual's or group's interactions affect their learning activities in a McGL environment.

As research on group learning has focused on the technological and pedagogical aspects, the social aspects of group learning remain under-investigated due to "the fact that the social aspects of collaboration are often taken for granted" [4]. While learning scientists have investigated learning and its processes, the mechanisms of social interaction in group learning are still not well understood. As a theory-driven framework, Engeström's [5] AST can enhance traditional qualitative investigations of complex learning environments, including McGL, by providing a well-defined framework and focus for analysis.

This paper proposes using AST as a method of sociocultural analysis to understand the complexity and inter-relatedness of McGL. AST focuses on the causal relationships between interacting components and their cultural determined mediators, with the goal of identifying conflicts and break-downs in learning using the construct of contradiction. Because most research using AST is qualitative in nature, the generalization of results is limited when conducting investigations of complex learning environments, nonetheless, it provides a rich and systematic description of the learning under study [2], [6], [7], [8], [20].

Engeström, following Leont'ev [15], proposed the activity as the unit of analysis because it fulfills the following requirements: "it is representative of the complexity of the whole, it is analyzable in its contextuality, it is specific to human beings by being culturally mediated, and it is dynamic rather than static" [9]. The activity in a group-learning situation, therefore, is represented in the multiple layers of the "construction and resolution of successively evolving tensions or contradictions in a complex system" [7].

In the following section of the paper, we discuss the phenomenon of group learning that comprises the McGL environment. It includes the conceptual definition of group learning, the paradigm shift of individual theories of learning to group or social theories of learning, and current trends of student mobility in higher education. We also argue that many constructivist approaches to learning tend to overemphasize the individual cognitive development instead of considering the situational and cultural factors involved in a successful group-learning experience. We then examine the sources of conflict in McGL by reviewing the different theoretical models of multiculturalism and the concept of diversity in relation to the grouplearning environment. Next, we present background information on Vygotsky's theoretical model and the work of post-Vygotskian CHAT scholars. Specifically, this paper discusses how Engeström's [5] AST can be used as a theoretical framework to better understand McGL interactions, including a review of studies that have relied on a CHAT-based analysis. The paper concludes with a discussion highlighting both advantages and criticisms of AST theoretically and methodologically, and potential research directions for studying McGL environments. 


\section{Group Learning}

Group learning has been defined as "a collection of persons who are emotionally, intellectually and aesthetically engaged in solving problems, creating products and meaning making - as assemblage in which each person learns autonomously and through the ways of learning of others" [4]. This definition includes several types of group learning that involve either face-to-face or online interactions with two or more participants such as collaborative, cooperative, team or transformative learning. Members of the group are expected to develop problem solving, interpersonal, and communication skills that are beneficial and meaningful for operating successfuly in a community. As group learning has been shown to be an effective approach in helping students to learn [1], [4] previous studies have addressed the dynamics of group learning to better understand decision-making behavior, group formation, adapting learning styles, group motivation, performance, group cohesion and cooperative structures. However, Hoover and Hashim [12] argued that it can only be considered to be group learning when the members see themselves as a unit or system, working with each other to achieve a common goal or understanding. The AST approach provides a means to systematically analyze students' interactions while considering how the individual's or group's interactions affect their learning in a McGL environment.

Many educational studies (e.g., [10], [11], [17], [20]) have emphasized the importance of developing deeper conceptual understanding as opposed to rote learning. Through group-based activities, members of the group are able to construct a deeper understanding of the subject matter and improve their thinking skills through thought-provoking multiple interpretations, and conflict negotiation. This interaction can only occur when two or more members of the group are engaged in dialogical processes, collectively building a body of knowledge and publically sharing their understanding. This coconstruction of knowledge depends on members of the group sharing a common understanding of performing tasks, achieving aims, establishing roles and developing rules through negotiation or dialog. However, this approach assumes that group members share a common understanding of these processes; this sharing becomes complex when there are differences in cultures, beliefs and practices among members of the group.

\section{Multiple Group Learning}

The transition from individual cognition to a group or social approach of learning has its roots in Hegel, who divided theories and philosophies of knowledge into two groups: individual-oriented approach and social-oriented approach [12]. The individual-oriented approach assumed that knowledge construction and mental representation are "developed in individual's mind, so that cognition and learning are still treated as a function of an individual mind" [17]. On the other hand, socialoriented theories emphasized that learning was the development of processes and relationships within the community in which individuals participated. Although both approaches are aimed at better understanding human learning, these forms of classification rely on two different epistemologies which determine how learning should be approached and analyzed.

Influenced by Descartes's works on the relationship between the mind and thinking processes, empiricism emphasized acquisition of knowledge based on an individual's mental reasoning which ultimately led to idea of instructionism in learning. Empiricists focused on observable content-based analysis for evidence of student learning. These approaches viewed education as a set of procedural rules that guided instructors to achieve well-defined student outcomes. The principles of instructionism developed through emphasis on teaching and learning-based rules, with the measurement of end results as evidence of student performance.

A different tack was taken by viewing cognition as the essential function of individual human learning. Kant argued that human understanding and evidence needed to be combined in order to determine whether learning had occurred. Kant's notion led to Piaget's model of constructivism in learning as Piaget believed that human learning consisted of learners actively constructing schemas in order to make sense of their world. Piaget's ideas shifted away from a purely empiricist approach by considering language, the real-world situation, and the development of cognitive abilities in the learner's construction of knowledge.

Vygotsky [19] argued that cognitive development in children might be limited at particular age, however, "with the help of social interaction, such as assistance from a mentor, students can comprehend concepts and schemas that they cannot know on their own." To better describe how learners acquired these concepts and schemas, Vygotsky [19] coined the term zone of proximal development (ZPD). ZPD is "the distance between the actual developmental level as determined by independent problem solving and the level of potential development as determined through problem solving under adult guidance or collaboration with more capable peers," (p. 86). Thus, it is not through individual mental activity that the learner acquires new schemas and concepts, but rather through social activity with mentors or teachers. 
Vygotsky emphasized that social interactions are the means of intellectual development through semiotic meditation within a specific sociocultural context. With a strong influence from Vygotsky's and his student Leont'ev's, work, Engeström [5] proposed AST as a descriptive theoretical framework for understanding the multiple interactions, dialog and conflicts (or contradictions) that arise in complex learning situations. To better understand group learning paradigm, Dillenbourg et al., [4] categorized three different theoretical perspectives within the Vygotskian tradition: the socio-constructivist, sociocultural and shared cognition approaches. The socialconstructivist approach as argues that "...it is above all through interacting with others, coordinating his or her approaches to the reality with those of others that the individual masters new approaches" [13].

Socio-cultural approaches focus on "the casual relationship between social interaction and individual cognitive change" [4], taking the basic unit of analysis as the social activity. Participants in a social activity may agree, argue and come to an "appropriation" [15], [16] with other members of the group until a shared understanding is achieved. However, as proposed by many sociocultural scholars these processes need to be verified through empirical studies and supported by comprehensive theoretical methods [3], [9], [11], [20]. The complexity of group learning becomes more sophisticated due to the mobility of diverse students, internationalization in higher education, and world economic policies. Thus, it increases the multicultural composition of group learning that then leads to the formation of McGL.

\subsection{Multicultural Dialog}

In most Vygotskian studies, the words culture and cultural have been associated with the words society, social and societal [3], [14] in order to provide a wider scope for research in areas of cultural diversity, ethnicity, social class and multiculturalism. Although, there is rarely consensus between North American and Russian scholars in interpreting the term social-cultural, Cole [3] has provided several definitions of culture that have been accepted and used by many sociocultural scholars.

Sociocultural scholars have seen culture as a bridge between members of the group and their environment [2], [11], [12], [14], [18]. The concept of culture helps members of the group construct a shared understanding through social interaction and negotiation. Göncü and Gauvain [10] defined culture as "a system of knowledge, beliefs, behaviors and customs that shared by members of an interacting group to which members can refer and that serve as the basic of further interaction" (p. 18). They also claimed that the shared social-cultural norms lead to co-construction of knowledge when members of the group are expected to participate in an adaptive process while negotiating differences. This resultant shared understanding of cultural differences, and newly negotiated norms, that develops over time, encourages a more successful group interaction that establishes a new set of rules for the sepcific social context.

\section{Engestrom's Activity System Theory}

Vygotsky proposed a theory of learning, introducing the concept of tool mediation (see Figure 1) to better describe the relationship between a human learner (the subject) and the target of learning (the object). The learning process is seen as a complex activity that is mediated using tools, situated within the learner's society or culture as it had evolved historically, and not simply within the individual mind [5], [6], [7], [14], [15]. The mediated activity could be established by watching, by listening or by becoming involved in a joint activity [8]. The Vygotskian model of tool-mediated activity focused on the use of cultural tools and signs, including language, as mediators in order to explain the semiotic processes that enable the development of human consciousness through participation in activities. Vygotsky also argued that psychologists needed to develop a unified framework that supports the objective study of human consciousness and therefore he was interested in identifying methods that could objectively study and explain human activities [5], [8], [15]. Vygotsky's mediated action triangle [19] consists of a subject (the learner), an object (the learning goal), and meditational artifacts or tools, which can be either concrete or symbolic. The components stand in dialectical relationship whereby each affects the others and the activity as a whole. The triangular representation of mediated activity is an attempt to explain human learning in a way that does not

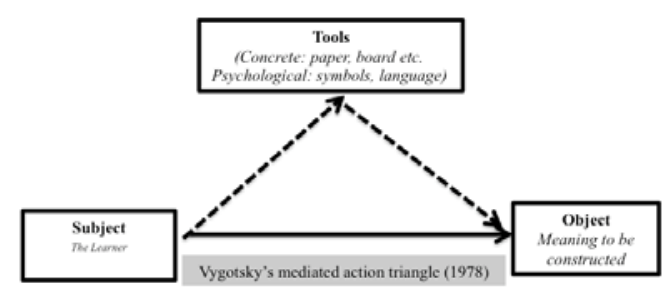

Figure 1. Vygotsky's mediated action triangle (1978)

rely on dualistic associations such as stimulusresponse [16]. The mediated activity involves both artifacts (concrete tools) and signs (psychological tools) to develop the formation of individual consciousness that helps the subject make meaning of the world [14], [15]. Vygotsky called this process, 
by which a learner (subject) constructs an understanding based on their experience with a social activity, internalization. However, the concept has been criticized because "it is based on a dualistic language, which is contradictory to how he explained mediated action and it can over simplify mediated action into an input and output process" [20]. By conceptualizing internalization as a theoretical construct within the Vygotskian model of toolmediated action, the study of activity became too person-centered and did not adequately address either the cultural dynamic, both of which are central to understanding McGL.

Due to the multiple interactions in McGL, we argue that Vygotsky's meditational model is not sufficiently elaborated to deal with the complexity of interactions, dialog, and problem-solving strategies that occur due to the learners' differences in culture, practices and beliefs. However, it is important to acknowledge that: (a) Vygotsky's meditational model is the basis of activity systems theory, and (b) the concept of activity from a CHAT perspective includes both observable experiences and mental activities [9], 18].

Leont'ev's second generation of activity theory moved beyond Vygotsky's individually focused to the community-based activity [15]. He provided a clear distinction between object-oriented activity and goal-directed actions. He concluded that "activity is thus not a reaction or a totality of reactions, but rather a system possessing structure, inner transformations, conversations, and development", (p. 10). Leont'ev's activity theory broadened the scope of Vygotsky's mediated action by introducing human activity as the unit of analysis to better understand the interaction among subject, object, motivation, action, goals, socio-historical context, and the consequences of the activity [7], [10], [15]. Leont'ev distinguished activity from actions and operations in that an activity involved the transformation of the object. Actions are conscious, tool-mediated and goal-oriented, whereas operations are routinized and therefore unconscious components of action subject to concrete conditions. He also conceptualized operations as "the methods for accomplishing actions" (p. 65). Activity, on the other hand, was distinguished by its object or purpose. Human activity, Leont'ev [15] argued, "does not exist except in the form of action or a chain of actions. If the actions that constitute activity are mentally subtracted from it then, absolutely nothing will be left of activity" (p. 64). Thus, an activity can always be defined uniquely by its object.

On the other hand, goal-directed actions are often seen as too person-focused and have less of a collective consequence to the community-based activity [15]. To better describe this communitybased activity, Engeström added rules, community and division of labor [5] to the bottom of Vygotksy's triangle (see Figure 2) as the socio-historical (cultural) components of mediated action in order "to move away from previous methods that were too person-focused or too goal-directed" [20]. The additional components guide the subjects on appropriate actions and acceptable interactions with other community members. The inclusion of cultural components in AST can serve as a framework for analysis that allows us to study the cultural issues (including conflicts and contradictions) in a natural way. AST thus provides us with a theoretical framework that already includes culture as an integral component of the learning activity.

From a psychological perspective, "the work of Leont'ev and his colleagues' focused on explaining and understanding how mental and observable activity can be regarded as a unit of analysis" [20], and how the interaction within an activity affects both the individuals and their environment. Leont'ev's

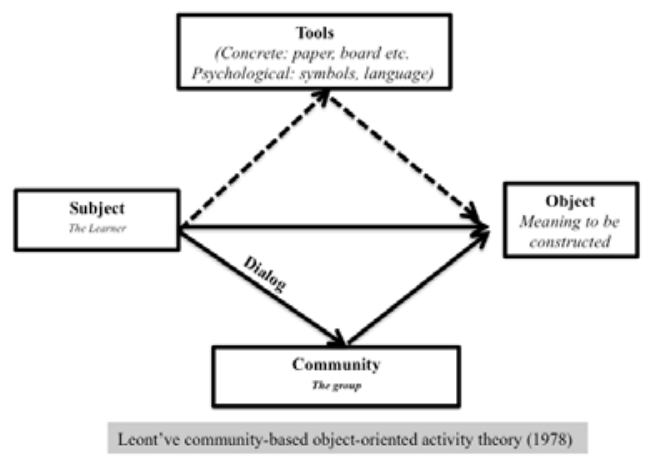

Figure 2. Leont've community-based object-oriented activity theory (1978)

mental and observable activity can be regarded as a unit of analysis" [20], and how the interaction within an activity affects both the individuals and their environment. Leont'ev's definition of activity and methodological approach (as a single unit of analysis) helps us to explain contradictions and the negotiation processes that occur among diverse learners (the individuals participants) in McGL situations (the environment). Rather than attempting to analyze McGL as a set of students each of whom is engaged in individual mental experiences, we can explain this complex learning as a series of objectoriented activities.

We argue that the learners' diverse backgrounds and the McGL environment cannot be treated or measured as isolated elements, rather they must be seen as inter-connected, and require a theoretical framework that permits such an analysis. Leont'ev [15] further argued that the meaning-making process includes both mental and physical representations of the activity when members of the group experience a 
series of negotiations in their goals and motives throughout the activity. Since the members of a McGL group come from different cultural groups, with different practices and beliefs, we further argue that the activity itself produces cultural formations within its own structures [3], [8], [9]. Once an activity is well negotiated and successfully accomplished, it becomes a robust and enduring tool that the group members can use in other learning activities.

Engeström [5], [6], [7], [8] built on Leont'ev [15] work in developing the concept of activity as a unit of analysis. Influenced by the Vygotskian model of tool-mediation action [19], Engeström's interpretation of activity as a unit of analysis fulfilled the following demands: "it is representative of the complexity of the whole, it is analyzable in its contextuality, it is specific to human beings by being culturally mediated, and it is dynamic rather than static" [9].

Engeström added the socio-historicalconstructs of rules, community and division of labor were to Vygotsky's [19] mediated action triangle. Engeström [5], [6] and Engeström and Miettinen [8] refer to rules as both formal or informal regulations that can, in fluctuating degree, constrain the activity and provide to the subject guidance in correct or expected procedures with acceptable interactions among members of the group community. Rules regulate the subject's actions toward an object and structure the social interaction of the group. Rules refer to the norms, routines, habits and values as well as unwritten or tacit practices. Community is described as a group of people who shared a common interest in carrying out an activity. In relation to the Vygotsky's mediated-tool triangle, the subject is part of a larger community, which conditions all the other components of the system. The division of labor within an activity includes "both the relatively horizontal division of tasks and the vertical division of power, positions, access to resources, and rewards" [9].

This expanded unit is referred as an activity system [3], [5], [6], which includes both Vygotsky's tool-mediated triangle and the socio-historical components. The AST triangle diagram (see Figure 3) depicts how the three central components (the subject, the community, and the object), and the three sets of mediators (tools, rules, and division of labor) interact and rely on each other.

We argue that the integration of the concept of activity as a system provides important advantages to the study of McGL, in that it analyzes group-based learning as a social activity by examining the interactions, dialog, and problem-solving strategies that arise from the learners' different cultures, practices and beliefs.

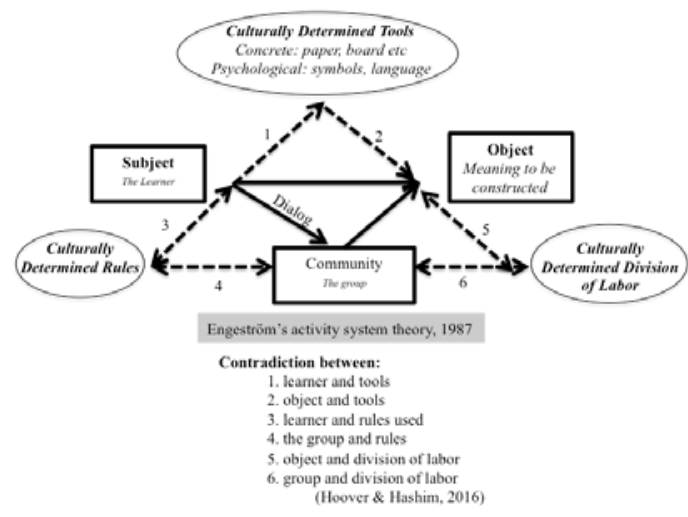

Figure 3. Multicultural group learning analysis based on Engeström's activity system theory (1987)

It enhances our understanding of human activity as situated in complex and collective learning situations [5]. The internal tensions and contradictions emerge and evolve within and between each of the three components (subject, object and community) and their mediators (tools, rules, and division of labor). This provides a powerful mechanism for describing the complex transitions and transformations that occur in McGL environments.

\section{A theoretical framework for identifying and analyzing contradictions in multicultural group learning}

The introduction of the third generation of activity theory [3], [5], [14] has sparked interest among scholars interested in analyzing learning interactions by identifying the activity as the unit of analysis [20]. However, as described above, such an activity can also trigger tensions caused by systemic contradictions especially when dealing with comples environments like McGL. In McGL, members of the group must negotiate the contradictions arising from different understandings and motives given their diverse social, cultural, and organizational backgrounds. These contradictions can directly affect the learner's ability to attain the object, or can even lead to the breakdown of the entire group activity.

However, the contradictions (conflicts and tensions) that arise in activity systems can be useful sources of analysis for better understanding of complex learning situations [8], [18], [20]. Engeström [5] argued that an activity system "is constantly working through contradictions within and between its elements. It is a virtual disturbance and innovation producing machine" (p. 11), that results in learning. In other words, it is the contradictions that reveal opportunities for creative innovations in problem-solving strategies, negotiation skills, structuring group dynamics and 
enacting the activity itself. The contradictions, conflicts, and discoordinations within an activity system provide a collective mirror for those involved in the activity, helping them to identify the sources of the problems, and can suggest potential avenues for expansive change [5], [6], [14]. AST best serves as a theoretical framework that allows researchers to better understand the cultural issues (including contradictions, conflicts or tensions) in a natural way. Since AST includes sociocultural components (tools, rules, and division of labor) as part of the learning activity, it permits easy identification and description of cultural contradictions.

In a McGL environment, with its diversity of participants, contradictions will occur in every collective activity and indicate emergent opportunities for expanding the activity. Since the individual's goal-directed actions are constantly subject to change, depending on the collective activity, a small change in any one element of the activity system can cause conflicts with other elements and place group members at cross-purposes [1], [10], [11]. Foot [9] identified three types of contradictions that can affect both the activity and group dynamics of complex learning environments:

a) contradictions between and among any of the elements of the activity system;

b) contradictions between and among activity systems; and

c) contradictions between and among participants from different activity systems that have different objects and motive (pp. 71- 73).

This typology can help to better explain the multiple layers of contradictions in McGL by providing a thorough analysis of the group-based interactions, dialog, and problem-solving strategies that can occur given the learners' differences in culture, practices and beliefs. It also provides a clear framework for how to analyze and identify themes in the data. It is important to understand that contradictions are not failures, but rather an inherent part of the process of carrying out an activity. In most group learning environments, contradictions and tensions occur when subjects modify and reconstruct activities while, at the same time, adapt to the current environment they are dealing with. As noted by Foot [9], "contradictions are not points of failure or deficits in the activity system in which they occur", (p. 66), rather, contradictions can serve as an impetus for modifying the activity by transforming the learners' understanding, learning and practices. Kuutti [13] argued that:

"Contradictions manifest themselves as problems, ruptures, breakdowns, and clashes. Activity theory sees contradictions as sources of development; activities are virtually always in the process of working through contradictions", (p. 34).

The evolution of an activity system occurs when members of community act to modify, adapt, resolve or transcend the system's contradictions. The process of identification and resolution of contradictions is a source of knowledge for co-construction that emerges in a pattern that Engeström [7] referred to as the 'expansive cycle' that reshapes the activity for the learners.

In McGL situations, the contradictions, conflicts and discoordinations provide opportunities for the activity's expansion because these disagreements provoke collective epistemic actions that lead to the co-construction of new knowledge among participants. The entire process of negotiating contradictions involves questioning, analyzing, modeling, examining, implementing, reflecting and consolidating an activity within a specific cultural context [8], [18], [20]. This negotiation of contradictions is a dynamic process that reflects the capacity of an activity to develop rather than simply function in a fixed and static mode.

However, contradictions that do not lead to negotiation may lead to failure of the activity. As the source for co-construction of knowledge, contradictions, tensions or disagreements should be understood as illuminative hinges that can open new vistas of understanding [9]. Contradictions, tensions or disagreements are not just obstacles to overcome or problems to be fixed in order to transform the object in achieving the desired learning outcome. Engeström [5] also associated the concept of contradictions with the concept of "zone of proximal development" [19] by linking the area between an activity system's present and foreseeable future, " [an]... area between actions embedded in the current activity with its historical roots and contradictions, the foreseeable activity in which the contradictions are expansively resolved, and the foreseeable activity in which the contradictions have led to contraction and destruction of opportunities" [7]. In other words, contradictions and tensions can encourage development, stunt development, or become the reason for co-construction of knowledge within complex learning environments.

In group learning environments, contradictions and tensions are resolved when subjects modify and re-construct new activities while, in the same time, adapting to the current environment. This cycle of learning actions provides potential avenues for expansive change in the learning activity. For example, in a McGL setting, participants with different backgrounds might question, critique or even reject the ideas presented due to the individual's speaking style. The term contradiction indicates "a misfit within elements [of an activity system], between them, between different activities, or 
between the different developmental phases of a single activity", [13]. By negotiating these contradictions, the participants can consolidate the outcomes into a new and stable form of practice or understanding. The process can go through several iterations until members of the group are satisfied with the desired outcome.

In some cases, as suggested by Yamagata-Lynch [20], the activity may break down completely and the subject may not be able to attain the object. By identifying the contradictions and understanding the process of negotiation as a source for co-construction of knowledge, we can better understand how cultural contradictions and tensions can drive transformations, which can result in the acquisition of schemas and skills which learners can then apply to future learning activities.

\section{Implications of AST for McGL}

The main advantage of using Engeström's AST in qualitative research is that this theory-based method can help researchers make sense of complex realworld and dynamic data sources in a manageable and meaningful manner. According to Barab et al., [2] Engeström [5], [6] and Yamagata-Lynch [20], AST provides a new method to extract meaningful information from massive and complex qualitative data sets without losing the richness of the data. In the context of McGL, an AST analysis helps the researcher conceptualize how multiple interactions can cause systemic contradictions within learning activities. An AST approach recognizes the complex nature and interaction of the elements in a learning situation, and provides a rigorous methodology for studying them without decomposing them into separate, isolated elements.

Importantly for the study of McGL, AST does not view cultural components as intrusions to the learning situation, but rather they are inherent to it. In the AST framework, members of the community are situated within a specific cultural context, and everything they do is shaped by and draws upon their own cultural values and resources. The term activity refers to what people do together, and is modified by the cultural context. Kozulin et al., [14] made this clear, "the issues of culture and learning have been inseparable for centuries for the simple reason that one of the main goals of learning is the transmission of culture from generation to generation" (p. 15). McGL produces new conflicts, contradictions, tensions, solutions, procedures, and systemic transformations in the group-learning environment.

AST provides a framework for describing and coding the contradictions that can arise in McGL environments. As can be seen in Figure 3, contradictions can be described as occurring between the three central components and their culturally determined mediators in six ways: between the (a) learner and tools (language and artifacts), (b) object and tools, (c) learner and rules used; (d) group and rules used, (e) group and the division of labor, and (f) object and the division of labor [12]. The greatest benefit of AST to researchers of multicultural group learning is the natural inclusion of culture in the mediators (tools, rules, division of labor) within a learning activity. Rather than seeing cultural issues as an intrusion into learning, AST sees them as inherent in the learning process. The tools, rules, and division of labors are necessarily culturally bound, even if the subject, community and object are not.

Engeström's AST can provide a richly descriptive answer to the question: Why and how diverse learners negotiate (or fail to negotiate) contradictions in McGL environment. Foot [9] noted, AST "is a heuristic framework for asking important questions that other theories may not address so clearly and for seeing relationships among those questions that may guide design and evaluation" (p. 66) for studies of complex learning environments. AST is based on three core assumptions: a) humans act collectively, learn by doing, and communicate in and through their actions, b) humans make, employ, and adapt tools of all kinds to learn and communicate, and c) the community is central to the process of making and interpreting meaning - and thus to all forms of learning, communicating, and acting. Learning activities are understood to be "a special kind of human activity developed in the course of societal development as an important aspect of human culture that has to be appropriated by individuals in order to be used, then, for concrete learning goals that depend on learning motives, objects and conditions" [11].

Based on this understanding, we argue that AST is the ideal theoretical framework for describing, understanding, and analyzing the complex interactions and relationships in McGL in which diverse learners are involved in a shared learning activity. As a method of analysis, Engeström [6] argued that it is "a strong candidate for such a unit of analysis in the concept of object-oriented, collective, and culturally mediated human activity, or activity system", (p. 9). The tensions and contradictions that might arise in an activity in McGL can be seen as a motive force of change and development that then produces the desired co-construction of knowledge.

Since McGL relies on members of a group who share a common understanding within a specific context of community (on tasks, aims, roles, rules, and tools) through negotiations or dialog [3], [5], [14], we claim that AST is the most comprehensive theory-driven framework to understand these multiple complex interactions. In many learning theories, individual action is regarded as the unit of analysis and the key to understanding human functioning. The orienting function of goals and plans, the sequential structure, and the levels of regulation of actions has received a great deal of 
attention. But these theories have difficulty in accounting for the socially distributed or collective aspects of learning, as well as the culturally mediated aspects of purposeful human behavior [7].

AST has much to contribute to the ongoing multidisciplinary field of interest in cultural practices and co-construction of knowledge. Activity system analysis is a "methodology that spawned from CHAT that can be valuable for qualitative researchers and practitioners who investigate issues related to real-world complex learning environment" [20]. AST further provides a rigorous and systematic framework for studying the influences of culture on student learning.

\section{Conclusion}

The number of international students in North American universities has increased significantly over the last several decades. Although both international and foreign students are expected to adapt to the local cultural norms of behavior and interaction, the extent to which these adaptations are happening remains unclear. This paper proposes using Engeström's Activity System Theory (AST) as a framework for the analysis of multicultural group learning (McGL). AST can best describe the realworld learning situation of multicultural group learning and identify the solutions resulting from students' negotiation of contradictions. By applying AST, researchers are able to identify and describe the contradictions that multicultural learners face, as well as the negotiation processes learners use to resolve them. As a first step, we propose a framework for analyzing contradictions in McGL as occurring between the three core components and their culturally determined mediators: (a) the learner and tools (language and artifact), (b) object and tools, (c) learner and rules used, (d) the group and rules used, (e) group and division of labor, and (f) object and division of labor.

The use of AST provides researchers with a rigorous, theory-based framework for identifying and analyzing the multiple interactions, dialog, and problem-solving strategies that occur in multicultural settings, in a way that naturally takes into account the learners' diversity of cultures, practices and beliefs.

\section{References}

[1] Astin, A., W. (1993). Diversity and multiculturalism on the campus: How are students affected?”, Change, 25(2), 44-49.

[2] Barab, S. A., Barnett, M., Yamagata-Lynch, L., Squire, K., \& Keating, T. (2002). Using activity theory to understand the systemic tensions characterizing a technology-rich introductory astronomy course. Mind, Culture,and Activity, 9(2),76. doi: 10.1207/S15327884 MCA0902_02.

[3] Cole, M. (1996). Cultural Psychology: A Once and Future Discipline. Cambridge, MA: Harvard University Press.

[4] Dillenbourg, P., Baker, M., Blaye, A. \& O'Malley, C. (1996). The evolution of research on collaborative learning. In E. Spada \& P. Reiman (Eds). Learning in Humans and Machine: Towards an interdisciplinary learning science. (pp. 189-211). Oxford: Elsevier.

[5] Engeström, Y. (1987). Learning by expanding: An activity-theoretical approach to developmental research. Helsinki: Orienta-Konsultit Oy. Retrieved November 30, 2016, from http://lchc.ucsd.edu/MCA/Paper/Engestrom/ expanding/toc.htm.

[6] Engeström, Y. (2000). Activity theory as a framework for analyzing and redesigning work. Ergonomics, 43(7), 960-974. doi: 10.1080/001401300409143.

[7] Engeström, Y. (2001). Expansive learning at work: Toward an activity theoretical conceptualization. Journal of Education and Work, 14(1), 133-156. doi: 10.1080/13639080020028747.

[8] Engeström, Y., \& Miettinen, R. (1999). Introduction. In Y. Engeström, R. Miettinen, \& R.-L. Punamaki (Eds.), Perspectives on activity theory (pp. 1-16). New York, NY: Cambridge University Press.

[9] Foot, K. A. (2001). Cultural-Historical Activity Theory as Practical Theory: Illuminating the Development of a Conflict Monitoring Network. Communication Theory, 11(1), 56-83.

[10] Göncü, A. \& Gauvain, M. (2010). Sociocultural Approaches to Educational Psychology: Theory, Research, and Application. in press, In K. Harris, J. Brophy., G. Sinatra, \& J. Sweller (Eds.). American Psychological Association, Educational Psychology Handbook: Contributions to Education. 1-56.

[11] Gurin, P., Dey, E. L., Hurtado, S., \& Gurin, G. (2002). Diversity and Higher Education: Theory and Impact on Educational Outcomes. Harvard Educational Review, 72 (3). 330-366.

[12] Hoover, M.L. \& Hashim, M.H. (2016). The Epistemology of Hegel: An Underlying Approach to Learning. International Journal for Cross-Disciplinary Subjects in Education 7 (3), 2830-2835. doi: DOI: 10.20533/ijcdse.2042.6364.2016.0385

[13] Kuutti, K. (1996). Activity theory as a potential framework for human-computer interaction research. In B. A. Nardi (Ed.), Context and consciousness (pp. 17-44). Cambridge, MA: MIT Press.

[14] Kozulin, A., Gindis, B., Ageyev, V., \& Miller, S. (2003). Vygotsky's educational theory in cultural context. Cambridge, England: Cambridge University Press. 
[15] Leont've, A. N. (1978). The problem of activity and psychology. In A. N. Leont'ev (Ed.), Activity, consciousness, and personality (pp. 45-74). Englewood Cliffs: Prentice Hall.

[16] Rogoff, B. (1990). Apprenticeship in thinking: Cognitive development in social context. New York: Oxford University Press.

[17] Sawyer, K. (2014). The new science of learning. In R. $\mathrm{K}$. Sawyer (Ed.). The Cambridge handbook of the learning sciences (pp. 1-18). NY: Cambridge University Press.

[18] Toomela, A. (2008). Vygotskian cultural-historical and sociocultural approaches represent two levels of analysis: Complementarity instead of opposition. Culture and Psychology, 14(1), 57-69. doi: 10.1177/1354067X07 085812.

[19] Vygotsky, L. S. (1978). Mind in society: The development of higher psychological processes. Cambridge, MA: Harvard University Press and Teacher Education, 19(6), 591-607. doi: 10.1016/S0742051X(03)00056-8.

[20] Yamagata-Lynch, L. C. (2010). Activity Systems Analysis Methods: Understanding Complex Learning Environments. New York: Springer. 